\title{
Effect of fiber length on thermomechanical properties of short carbon fiber reinforced polypropylene composites
}

\begin{abstract}
Carbon fiber reinforced composites have all the ideal properties, leading to their rapid development and successful use for many applications over the last decade. In this paper, short carbon fiber reinforced polypropylene (SCF/PP) composite were prepared with melt blending and hot-pressing techniques. The thermomechanical properties of this composite were investigated taking into account the combined effect of mean fiber length. Thermal stability of the composite was studied via the thermal gravimetric analysis (TGA) and dynamic mechanical analysis (DMA) was used to measure the damping properties of the composites. Finally it can be shown that an increase in fiber length can enhance the thermal stability of SCF/PP composites and improve the damping properties as well.
\end{abstract}

Keyword: A. Polypropylene; A. Thermoplastic; A. Composites; B. Carbon fibers; G. Thermogravimetric analysis (TGA, G. Dynamic mechanical analysis (DMA) 